\title{
ESTADO TAXONÓMICO DE ICHTHYOCEPHALUS ANAFE GARCÍA ET COY, 1996 E I. GUANIGUANICO GARCÍA ET COY, 1999 \\ (RHIGONEMATIDA: ICHTHYOCEPHALIDAE) \\ Y NUEVOS REGISTROS DE I. CUBENSIS SPIRIDONOV, 1989 PARA CUBA
}

\author{
Nayla García Rodríguez y Jans Morffe Rodríguez
}

Instituto de Ecología y Sistemática. Carretera Varona 11835 e/ Oriente y Lindero, La Habana 19, CP

11900, Calabazar, Boyeros, La Habana, Cuba.nayla@ecologia.cu, jans@ecologia.cu

\section{RESUMEN}

Se discute el estado taxonómico de Ichthyocephalus anafe García et Coy, 1996 e I. guaniguanico Coy et García, 1999 (Nematoda: Ichthyocephalidae). La revisión de material tipo de I. anafe, así como de nuevos ejemplares provenientes de la localidad y hospedero tipos de I. guaniguanico demostró que ambas especies presentan similitudes morfométricas y morfológicas con I. cubensis Spiridonov, 1989. De esta forma, I. anafe e I. guaniguanico se consideran sinónimos de I. cubensis. Se registra la especie para dos nuevas localidades de Cuba: Tres Ceibas de Clavellinas, provincia de Matanzas y Jobo Rosado, provincia de Sancti Spíritus.

Palabras clave: Nematoda, Ichthyocephalus, sinonimia, Diplopoda, Cuba.

Title: Taxonomical status of Ichthyocephalus anafe García \& Coy, 1996 and I. guaniguanico García \& Coy, 1999 (Rhigonematida: Ichthyocephalidae) and new records of I. cubensis Spiridonov, 1989 from Cuba.

\section{ABSTRACT}

The taxonomical status of Ichthyocephalus anafe García \& Coy, 1996 and I. guaniguanico Coy \& García, 1999 (Nematoda: Ichthyocephalidae) is discussed. The revision of types of I. anafe as well as new material from the type locality and host of I. guaniguanico showed the existence of morphometrical and morphological similarities of the latter taxa with I. cubensis Spiridonov, 1989. Therefore, I. anafe and I. guaniguanico are considered synonyms of I. cubensis. The species is recorded for two new localities from Cuba: Tres Ceibas de Clavellinas, Matanzas province and Jobo Rosado, Sancti Spíritus province.

Keywords: Nematoda, Ichthyocephalus, synonymy, Diplopoda, Cuba.

\section{INTRODUCCIÓN}

La familia Ichthyocephalidae Travassos et Kloss, 1958 (Nematoda: Rhigonematida) incluye a los géneros Ichthyocephalus Artigas, 1926; Paraichthyocephalus Travassos et Kloss, 1958 e Ichthyocephaloides Hunt et Sutherland, 1984 (Artigas, 1926; Hunt y Sutherland, 1984; Travassos y Kloss, 1958). Estos se caracterizan por la apertura oral transversal, lateralmente orientada, formada por un labio dorsal y uno ventral, lo que unido a su cápsula cefálica esclerotizada y sus cuatro papilas cónicas le brindan una apariencia muy peculiar.

Del género Ichthyocephalus se conocen 15 especies, cuatro de ellas registradas para Cuba. Spiridonov (1989) describe la primera de ellas, I. cubensis Spiridonov, 1989, parásita de Rhinocricus sp. (Diplopoda: Rhinocricidae), en la región oriental. Coy et al. (1993) registran a 
I. cubensis Coy, García et Álvarez, 1993 como nueva especie para el occidente. Esta homonimia es resuelta por Coy y García (1999), renombrando a la especie como I. guaniguanico Coy et García, 1999.

García y Coy $(1995,1996)$ describen a I. spinosus García et Coy, 1995 e I. anafe García et Coy, 1996 de las Alturas de Trinidad y la Sierra de Anafe, respectivamente. Todas las especies descritas para Cuba son parásitas de espirobólidos de la familia Rhinocricidae (Diplopoda: Spirobolida) y junto a las de Guadalupe, Puerto Rico y La Española, son las únicas del género en presentar divertículo vaginal.

El reexamen del material tipo depositado en colecciones correspondiente a I. anafe y nuevo material procedente de la localidad y hospedante tipos de I. guaniguanico, así como de otras dos nuevas localidades, permitió establecer la ausencia de diferencias morfológicas y morfométricas entre estas especies por lo que las mismas se consideran sinónimos de I. cubensis.

\section{OBJETIVOS}

- Esclarecer el estado taxonómico de I. anafe e I. guaniguanico.

- Registrar a I. cubensis para las nuevas localidades de Tres Ceibas de Clavellinas, provincia de Matanzas y Jobo Rosado, provincia de Sancti Spíritus.

\section{MATERIALES Y MÉTODOS}

Se recolectaron manualmente varios ejemplares de Rhinocricus duvernoyi (Diplopoda: Rhinocricidae), bajo hojarasca en El Salón, Sierra del Rosario, provincia de Artemisa, Cuba (un ejemplar), Tres Ceibas de Clavellinas, Limonar, provincia de Matanzas, Cuba (dos ejemplares) y Jobo Rosado, Yaguajay, provincia de Sancti Spíritus, Cuba (cinco ejemplares). Los diplópodos se conservaron vivos en recipientes plásticos con tapa agujereada y hojarasca como fuente de alimento y humedad, hasta el momento de ser procesados.

Los hospederos se sacrificaron con vapores de éter etílico o de acetona y sus intestinos se extrajeron inmediatamente, mediante cortes en los segmentos posteriores del cuerpo. Dichos intestinos se disecaron en solución salina fisiológica ( $\mathrm{NaCl}$ al $0.9 \%)$. Los nemátodos se sacrificaron en solución salina caliente $\left(70^{\circ} \mathrm{C}\right)$ y se fijaron en etanol al $70 \%$.

Los nemátodos se transfirieron a glicerina anhidra según el método de Seinhorst (1959) y se montaron en el mismo medio. Los bordes de los cubreobjetos se sellaron con esmalte de uñas para prevenir la hidratación de la glicerina. Las medidas tomadas fueron las propuestas por Hunt (1999) y se realizaron con ayuda de un micrómetro ocular $( \pm 0.001 \mathrm{~mm})$. Las mismas están expresadas en milímetros. Se calcularon, además, los índices de De Man a, b, c y V. De cada variable se ofrece su rango, seguido por la media, la desviación estándar y el número de mediciones, entre paréntesis.

Algunos ejemplares se procesaron para Microscopía Electrónica de Barrido: se deshidrataron en una serie de etanol de concentración creciente, se secaron hasta punto crítico mediante $\mathrm{CO}_{2}$ líquido y se cubrieron de oro. Las microfotografías se realizaron a un voltaje de $22 \mathrm{kV}$.

Los dibujos a línea se realizaron mediante los programas CorelDRAW X3 y Adobe Photoshop CS2, sobre la base de microfotografías tomadas con una cámara digital AxioCam acoplada a un microscopio Carl Zeiss AxiosKop 2 Plus. Las escalas de todas las ilustraciones están dadas en milímetros. 
El material estudiado se encuentra depositado en la Colección Helmintológica de las Colecciones Zoológicas del Instituto de Ecología y Sistemática (CZACC), La Habana, Cuba; la Coleçao Helmintologica do Instituto Oswaldo Cruz (CHIOC), Río de Janeiro, Brasil y la Colección Nematológica del Royal Belgian Institute of Natural Sciences (RIT), Bruselas, Bélgica.

\section{RESULTADOS}

SISTEMÁTICA

Familia Ichthyocephalidae Travassos et Kloss, 1958

Ichthyocephalus Artigas, 1926

Ichthyocephalus cubensis Spiridonov, 1989

Figuras 1, A-I y 2, A-F

Ichthyocephalus cubensis Spiridonov, 1989:77-79, Fig. 3, A-B; pl. V

Ichthyocephalus cubensis Coy, García y Álvarez, 1993:33-35, Fig. 1, homonimia

Ichthyocephalus guaniguanico Coy y García, 1999:8-9, sinonimia

Ichthyocephalus anafe García y Coy, 1996:90-91, Fig.1, A-E, sinonimia

Material examinado. Tres (3) §ð, parátipos de Ichthyocephalus anafe; Cuba, Artemisa, Caimito, Mesa de Anafe; en Rhinocricus duvernoyi; III/1993; A. Juarrero y L. M. Hernández col.; CZACC 11.4261-4263. 3 우, igual información que los anteriores; CZACC 11.4264-11.4266.

Cinco (5) $q$ q de Ichthyocephalus cubensis; Cuba, Artemisa, Candelaria, Sierra del Rosario, El Salón; en Rhinocricus duvernoyi; 2012; E. Fonseca col.; CZACC 11.4818-11.4822.9 §ત, igual información que los anteriores; CZACC 11.4823-11.4831.

Nueve (9) 9 de Ichthyocephalus cubensis; Cuba, Sancti Spíritus, Yaguajay, Jobo Rosado;

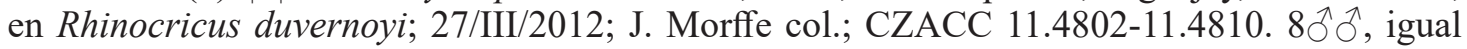
información que los anteriores; CZACC 11.4811-11.4818. 2우, igual información que los anteriores; RIT. $2 \hat{\jmath}$, igual información que los anteriores; RIT. $q$, igual información que los anteriores; CHIOC. $\hat{\jmath}$, igual información que los anteriores; CHIOC.

Cuatro (4) 90 de Ichthyocephalus cubensis; Cuba, Matanzas, Limonar, Tres Ceibas de Clavellinas; en Rhinocricus duvernoyi; VII/2011; D. Saladrigas col.; CZACC 11.4793-11.4796. $5 \hat{\jmath} \widehat{\jmath}$, igual información que los anteriores; CZACC 11.4797-11.4801.

Discusión. Spiridonov (1989), describe a I. cubensis a partir de la forma y tamaño de sus espículas, elongadas y marcadamente desiguales (espícula derecha 0.650-0.820, espícula izquierda 0.230-0.260), así como la forma del borde de la región cefálica. Coy et al. (1993) registran a $I$. cubensis como nueva especie, teniendo en cuenta su gran talla ( $\lesssim 10.200-12.700$, \$13.400-18.500) y la presencia de un istmo muy reducido, además del tamaño de sus espículas (espícula derecha 0.680-0.910, espícula izquierda 0.221-0.247), desconociendo la especie de igual nombre descrita por Spiridonov (1989).

García y Coy (1995) describen otra nueva especie, I. spinosus, teniendo en cuenta la presencia de microtricas en toda la superficie corporal, incluido el extremo apical de la cola. 

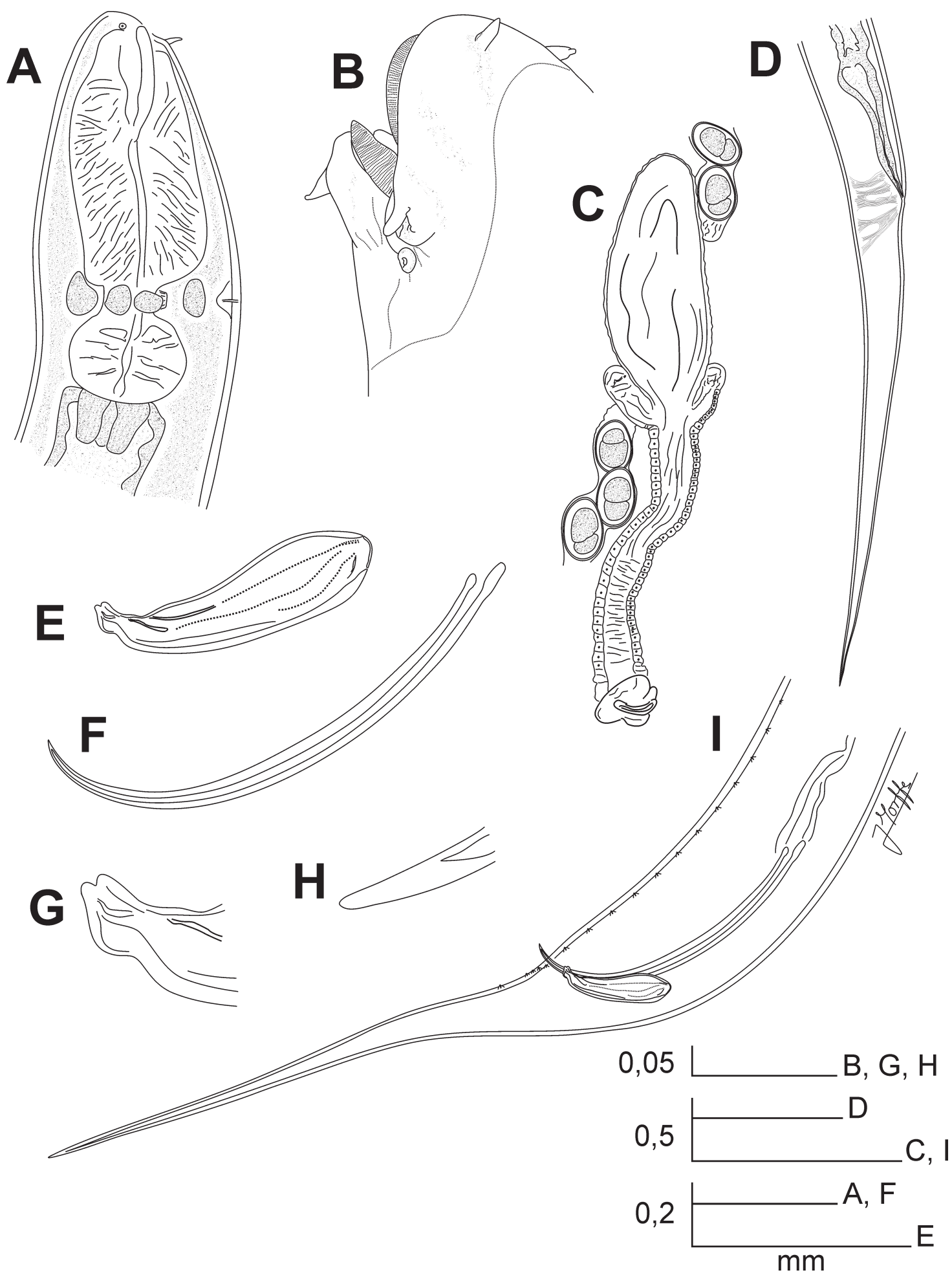

Figura 1. Ichthyocephalus cubensis Spiridonov, 1989. Hembra. A. Región esofágica, vista lateral. B. Extremo cefálico, vista dorsolateral. C. Vulva y divertículo vaginal. D. Cola, vista lateral. Macho. E. Espícula izquierda. F. Espícula derecha. G. Extremo distal de la espícula izquierda. H. Extremo distal de la espícula derecha. I. Cola, vista lateral. 

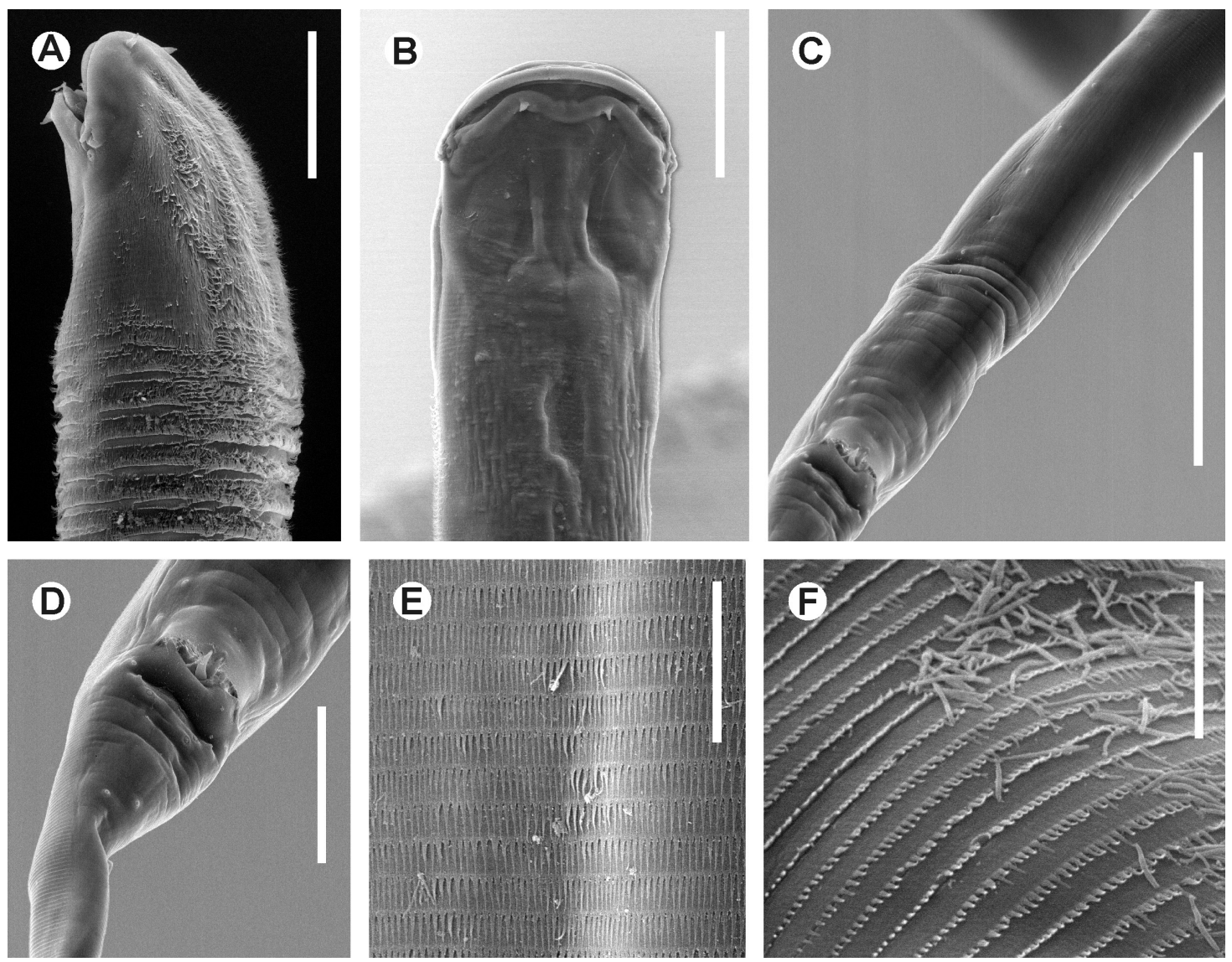

Figura 2. Ichthyocephalus cubensis Spiridonov, 1989. Microfotografías al Microscopio Electrónico de Barrido. A. Región cefálica de la hembra, vista lateral. B. Región cefálica de la hembra, vista ventral. C. Región caudal del macho, vista ventral. D. Región cloacal del macho, vista ventral. E. Microtricas de la región media del cuerpo. F. Microtricas de la región caudal. Líneas de escala: A, B, D. 0.10 mm.C. $0.30 \mathrm{~mm}$. E. $0.025 \mathrm{~mm}$. F. $0.010 \mathrm{~mm}$.

I. spinosus presenta además, marcadas diferencias de talla con I. cubensis de Coy et al. (1993) (万3.354-4.160 vs. 10.200-12.700, $94.290-6.760$ vs. 13.400-18.500) e igual sucede con el resto de sus caracteres morfométricos.

La especie I. anafe se describe como muy similar a I. cubensis por su morfometría y aspecto general. Las diferencias entre ambas especies son establecidas a partir de la longitud del cuerpo esofágico (0.399-0.430 vs. 0.280-0.320), y el diámetro del bulbo (0.105-0.178 vs. 0.143-0.208) de las hembras y la cola en los machos (1.102 vs. 0.680-0.910) además de la relación espicular 4:1, con la espícula derecha algo más larga en I. anafe (0.892-0.913 vs. 0.680-0.910). También el patrón papilar con 12 pares de papilas preanales y dos postanales en $I$. anafe y de 8 a 10 pares preanales y de 2 a 3 pares postanales en I. cubensis (Coy et al., 1993; García y Coy, 1996).

Coy y García (1999), renombran a la especie de Coy et al. (1993) como I. guaniguanico, dando solución a la homonimia anteriormente provocada. Estos autores establecen las diferencias entre estas especies por el tamaño y extensión de las microtricas, de mayor talla y restringidas a la región cervical en $I$. guaniguanico y la presencia de divertículo vaginal en las hembras. En I. cubensis las microtricas están presentes en todo el cuerpo y el divertículo vaginal está ausente, según Spiridonov (1989). 
Hunt (1999) redescribe detalladamente a I. cubensis, añadiendo las observaciones hechas a partir de la Microscopía Electrónica de Barrido, enmarcando sus diferencias con el resto de las especies del género por su gran talla y la pronunciada desigualdad de sus espículas, su supernumerario y asimétrico patrón papilar (de 31 a 38 papilas, con 11-13-14 pares preanales y 5-6 postanales) y la extensión y forma de sus microtricas. Incluye la presencia de un divertículo vaginal, carácter este no reflejado en la descripción original y presente en el resto de las especies de Ichthyocephalus registrados para Cuba, además de I. anadenoboli Van Waerebeke, Adamson et Kermarrec, 1984 de Guadalupe (Van Waerebeke et al., 1984). I. alayoi García, Coy et Ventosa, 2001 e I. victori García et Fontenla, 2002; de La Española y Puerto Rico, respectivamente, también presentan este último carácter (García et al., 2001; García y Fontenla, 2002).

El reexamen del material tipo depositado en colecciones correspondiente a I. anafe y nuevos ejemplares procedentes de la localidad y hospedante tipos de I. guaniguanico, permitió establecer que las diferencias morfométricas y morfológicas observadas entre estas especies, se encontraban dentro del rango de variación de I. cubensis, según la redescripcion de Hunt (1999), incluida la forma y extensión de las microtricas, el patrón papilar y la pronunciada desigualdad de las espículas. Los ejemplares asignados a ambas especies, presentan la cutícula finamente estriada, totalmente cubierta de microtricas densas, más largas y algo curvadas en la región anterior y más cortas y rectas en el resto del cuerpo (Fig. 2, A, E y F).

Los machos asignados a estas dos especies presentan de 11 a 14 pares de papilas preanales y 5 a 6 postanales, con el característico patrón asimétrico y supernumerario de I. cubensis (Fig. 2, C-D). La longitud de las espículas de I. anafe (espícula derecha 0.710-0.760, espícula izquierda 0.240-0.260) e I. guaniguanico (espícula derecha 0.710-0.950, espícula izquierda 0.230-0.270), aunque algo menores como promedio, se encuentran en el rango de variación de $I$. cubensis (espícula derecha 0.748-0.953, espícula izquierda 0.229-0.269). No se aprecian diferencias notables en el resto de los caracteres morfométricos, con excepción de la talla algo superior en los machos correspondientes a I. guaniguanico (12.400-13.900 vs. 9.970-12.390), una mayor anchura (0.350-0.475 vs. 0.248-0.361) y un esófago algo más corto $(0.420-0.520$ vs. 0.5860.666). Igual sucede con el ancho del cuerpo (0.360-0.380 vs. 0.248-0.361) y la longitud del esófago (0.490-0.510 vs. 0.586-0.666) en I. anafe (Tabla I).

Las hembras sólo difieren levemente en la longitud del cuerpo (15.725-18.275 vs. 13.61016.810) y la anchura máxima (0.390-0.510 vs. 0.281-0.374), algo superiores, así como en la longitud del esófago (0.470-0.550 vs. 0.642-0.792), menor en I. guaniguanico. Algo similar ocurre con el ancho máximo (0.380-0.490 vs. 0.281-0.374) y la longitud del esófago (0.5200.550 vs. 0.642-0.792) en I. anafe (Tabla II).

Se considera entonces que las especies I. guaniguanico e I. anafe constituyen sinónimos de I. cubensis. Los ejemplares de las poblaciones de Tres Ceibas de Clavellinas, Matanzas y Jobo Rosado, Sancti Spíritus se corresponden métrica y morfológicamente con los caracteres descritos para I. cubensis. Las diferencias morfométricas coinciden con las descritas para El Salón (I. guaniguanico) y Sierra de Anafe (I. anafe) en cuanto a la talla, anchura máxima y longitud del esófago en ambos sexos y la longitud de la cola en las hembras y de las espículas en los machos (Tablas I y II). Ambas poblaciones constituyen nuevos registros de localidad para la especie. 


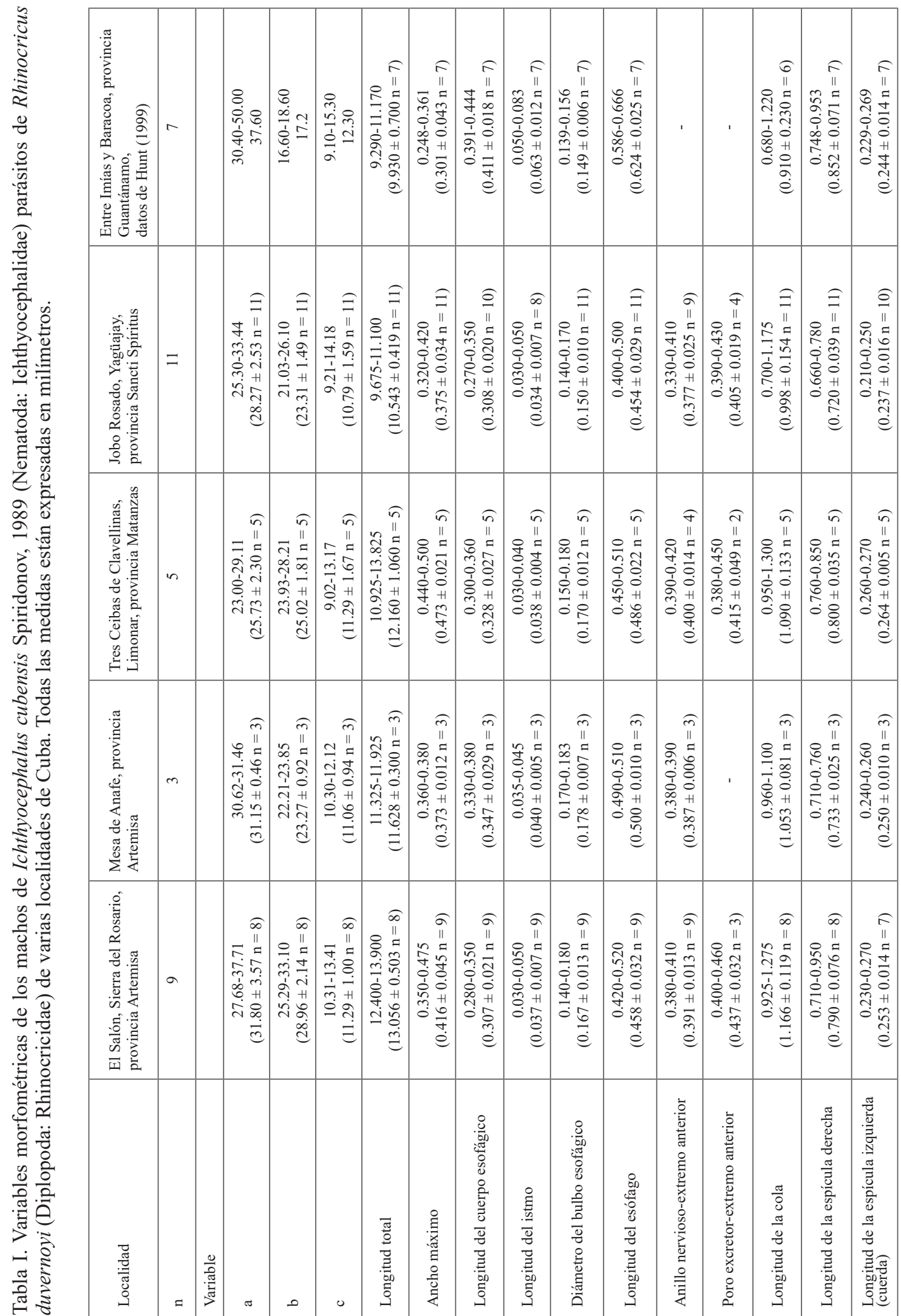




\begin{tabular}{|c|c|c|c|c|c|c|c|c|c|c|c|c|c|c|c|c|c|}
\hline 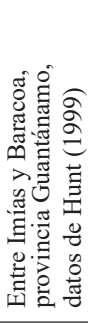 & 0 & & 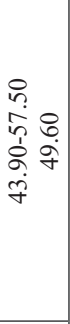 & 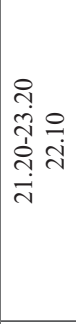 & 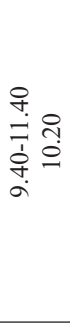 & 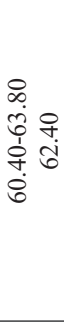 & 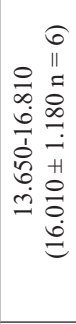 & 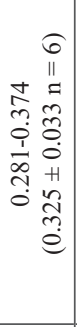 & 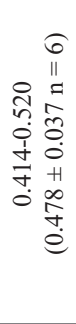 & 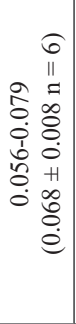 & 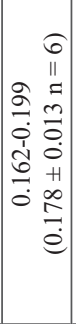 & 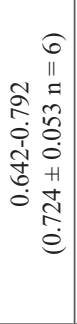 & & & & 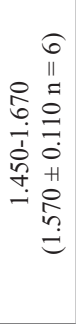 & 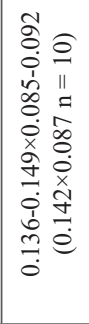 \\
\hline 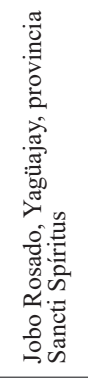 & $\simeq$ & & 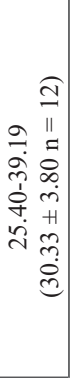 & 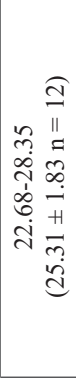 & 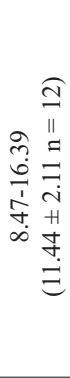 & 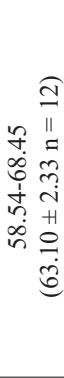 & 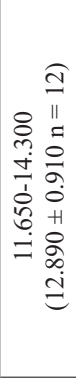 & 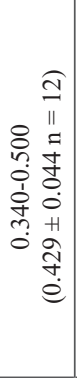 & 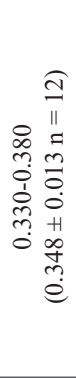 & 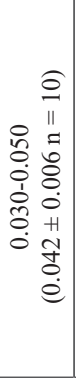 & 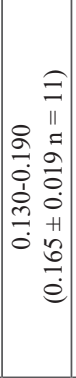 & 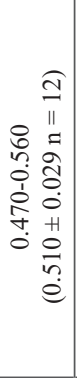 & 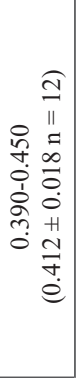 & 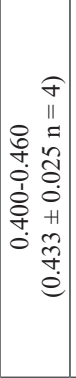 & 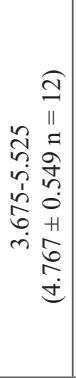 & 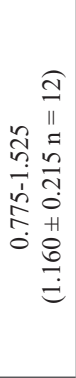 & 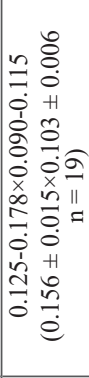 \\
\hline 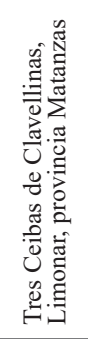 & $\nabla$ & & 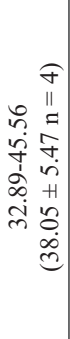 & 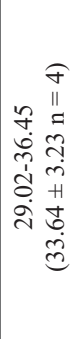 & 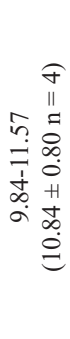 & 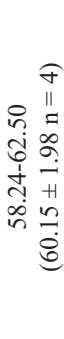 & 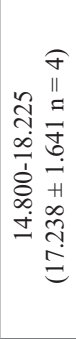 & 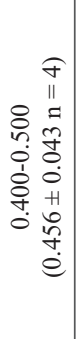 & 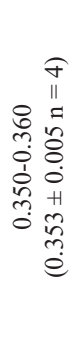 & 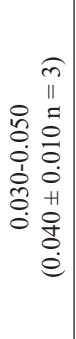 & 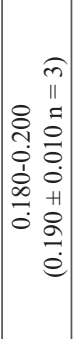 & 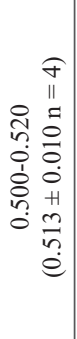 & 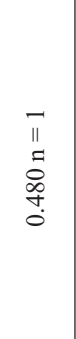 & 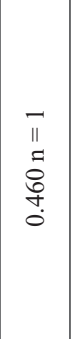 & 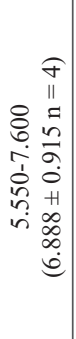 & 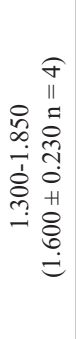 & 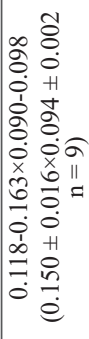 \\
\hline 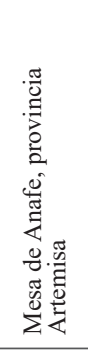 & $m$ & & 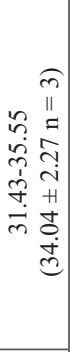 & 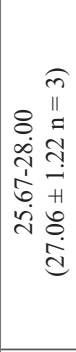 & 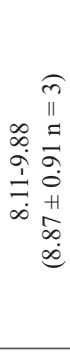 & 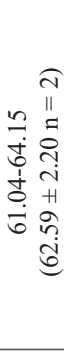 & 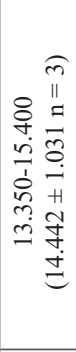 & 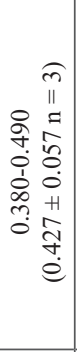 & 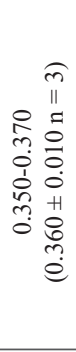 & 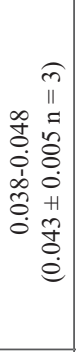 & 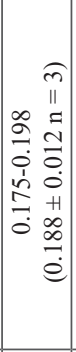 & 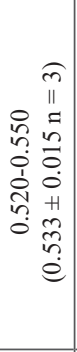 & 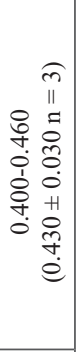 & ' & 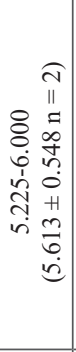 & 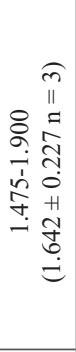 & 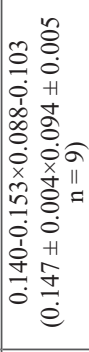 \\
\hline 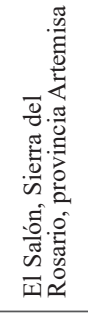 & in & & 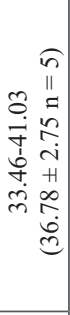 & 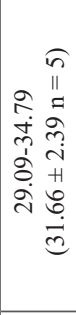 & 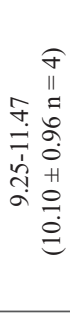 & 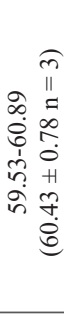 & 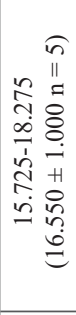 & 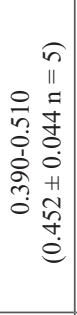 & 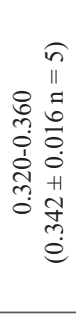 & 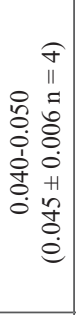 & 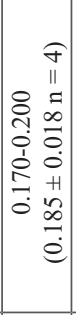 & 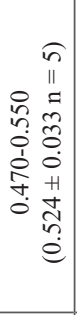 & 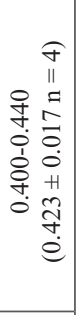 & $\begin{array}{l}\text { N } \\
\| \\
\stackrel{7}{0} \\
\stackrel{\overbrace{}}{+} \\
\stackrel{0}{0}\end{array}$ & 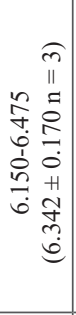 & 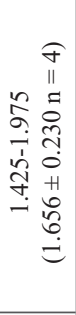 & 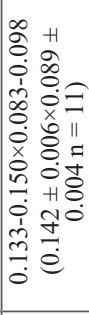 \\
\hline 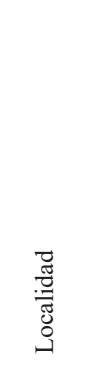 & $\Rightarrow$ & 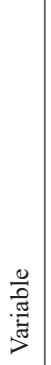 & $\approx$ & م & 0 & $>$ & 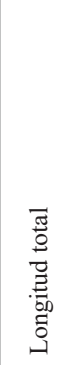 & 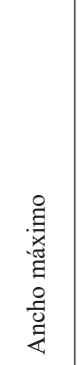 & 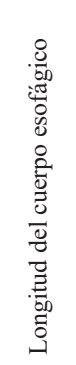 & 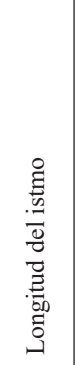 & 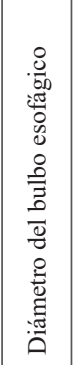 & 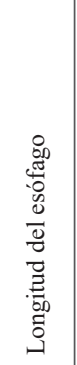 & 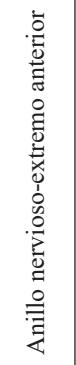 & 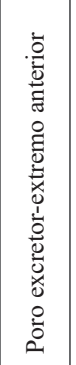 & 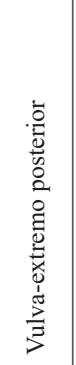 & 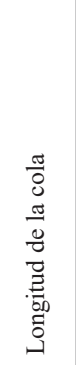 & 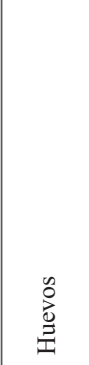 \\
\hline
\end{tabular}




\section{AGRADECIMIENTOS}

Al MSc. Yamir Torres y MSc. Eduardo Furrazola, del Instituto de Ecología y Sistemática por su ayuda con las microfotografías. A Julien Cillis del Royal Belgian Institute of Natural Sciences por su asistencia técnica con el Microscopio Electrónico de Barrido. A la Dra. Wilfrida Decraemer y la Dra. Marie-Lucie Susini, del Royal Belgian Institute of Natural Sciences, por su ayuda durante la estancia del segundo autor en dicha institución. Al Dr. Luis F. De Armas del Instituto de Ecología y Sistemática por la revisión del manuscrito. El acceso a técnicas de Microscopía Electrónica de Barrido fue financiado por el Belgian Development Cooperation a través del Belgian Focal Point of the Global Taxonomy Initiative (GTI), en su edición del 2012, otorgado al segundo autor. A IDEAWILD por su donativo para el trabajo de campo y laboratorio. Estos resultados están inscritos en el proyecto PP-02 "Curaduría de Colecciones Zoológicas" del Instituto de Ecología y Sistemática.

\section{LITERATURA CITADA}

Artigas, P. 1926. Nematoideos de invertebrados (III). Boletim Biologico, 1: 59-71.

Coy, A. y N. García. 1999. Un caso de homonimia en rigonemátidos cubanos (Nematoda; Rhigonematida). Cocuyo, 9: 8-9.

Coy, A., N. García y M. Alvarez. 1993. Nemátodos parásitos de diplópodos cubanos con descripción de nueve especies, siete de ellas nuevas. Acta Biológica Venezolana, 14: 33-51.

García, N. y A. Coy. 1995. Nuevas especies de nemátodos (Nematoda) parásitos de artrópodos cubanos. Avicennia, 3: 87-96.

García, N. y A. Coy. 1996. Nemátodos de Artrópodos de la Sierra de Anafe, Cuba. Avicennia, 4/5: 89-94.

García, N., A. Coy y L. Ventosa. 2001. Tres especies nuevas de rigonemátidos (Nematoda: Rhigonematida), parásitos de diplópodos (Diplopoda: Spirobolida) de La Española. Solenodon, 1: 25-32.

García, N. y J. L. Fontenla. 2002. New species of nematodes (Nematoda: Rhigonematida) associated with Rhinocricus parcus (Diplopoda) from Puerto Rico. Solenodon, 2: 1-5.

Hunt, D. J. 1999. The Ichthyocephalidae (Nematoda: Rhigonematida). Characterisation and morphology with proposal of two new species of Ichthyocephaloides and additional data on Ichthyocephalus cubensis and I. anadenoboli International Journal of Nematology, 9: 101-118.

Hunt, D. J. y J. Sutherland. 1984. Ichthyocephaloides dasyacanthus n.g., n.sp. (Nematoda: Rhigonematoidea) from a millipede from Papua New Guinea. Systematic Parasitology, 6: 141-146.

Seinhorst, J. W. 1959. A rapid method for the transfer of nematodes from fixative to anhydrous glycerin. Nematologica, 4: 67-69.

Spiridonov, S. E. 1989. New species of Rhigonematida (Nematoda) from the Cuban spirobolid Rhinocricus sp. (Diplopoda) Folia Parasitologica, 36: 71-82. 
Travassos, L. y G. R. Kloss. 1958. Ichthyocephalidae familia nova (Nematoda). Boletim do Museu Paraense Emilio Goeldi, 17: 1-19.

Van Waerebeke, D., M. L. Adamson y A. Kermarrec. 1984. Spermiogénèse et fonction du sac vaginal chez Ichthyocephalus anadenoboli n. sp. (Rhigonematidae; Nematoda), parasite d'Anadenobolus politus (Porat) (Rhinocricidae; Diplopoda) en GuadeloupeAnnales de Parasitologie Humaine et Comparée, 59: 101-109.

[Recibido: 02 de junio, 2014. Aceptado para publicación: 01 de septiembre, 2014] 